\title{
Influence of soft interlayers on fretting fatigue and fretting wear resistance of Ti-6Al-4V alloy
}

\author{
Amin Ma, Daoxin Liu*,Xiaohua Zhang
}

Institute of Corrosion and Protection, School of Aeronautics, Northwestern Polytechnical University, Xi'an, Shaanxi, 710072, China

Corresponding author.

E-mail address: liudaox@nwpu.edu.cn (Daoxin Liu).

\begin{abstract}
Ti-6Al-4V alloy is the main structure material of aerospace components due to its excellent corrosion resistance, high specific strength and other good characteristics. However, this alloy has low hardness, poor wear resistance and higher friction coefficient, so it is very sensitive to fretting wear (FW) and fretting fatigue (FF) damages. In this work, we studied three kinds of soft inter-layers (copper foil, nickel foil and polytetrafluoroethylene (PTFE) lamella) between the Ti-6Al-4V fatigue samples and the Ti-6Al-4V counterparts respectively to enhance the FF and FW resistance of this alloy. The results show that the method is an economical and effective way to increase the FF and FW resistance of Ti-6Al-4V alloy. The order of enhancement in the FF resistance of the titanium alloy was copper foil $>$ PTFE lamella $>$ nickel foil. Additionally, the friction coefficients of PTFE lamella, copper foil, nickel foil and Ti-6Al-4V alloy were about 0.10, 0.60, 0.65 and 1.1, respectively. The lowest friction coefficient of PTFE, compared with copper foil, nickel foil and substrate, was of great benefit to improving the FF property of Ti-6Al-4V alloy. Nevertheless, the FF life of Ti-6Al-4V alloy with the copper interlayer was higher than PTFE, which was about 5 times higher than substrate.
\end{abstract}

Keywords: Soft foil interlayer, Fretting fatigue, Fretting wear, Ti-6Al-4V alloy

\section{Introduction}

The Ti-6Al-4V alloy is widely used in aerospace field due to their high strength/weight ratio, corrosion resistance, thermal stability properties, and other excellent comprehensive performance. However, this alloy is very sensitive to damage from fretting wear (FW) and fretting fatigue (FF) because of its low thermal conductivity and poor wear resistance, which would influence the security and service life of aeronautic equipment $[1-3]$. The mechanism of fretting fatigue damage is complex, it is a synergistic effect between fretting wear and fatigue damage. Thus, the way to improve the fretting wear resistance of materials could also improve the fretting fatigue resistance of this alloy to some extent $[\underline{4}, \underline{5}]$. The way to improve the FF and FW properties of Ti-6Al-4V alloy is still a hot research area.

The hard coatings of $\mathrm{CrCN}$ and TiAlN were used to improve the wear resistance of Ti-6Al-4V alloy successfully by Hager [6]. Additionally, Zhang [7] prepared a lubrication film of $\mathrm{Cu} / \mathrm{Ni}$ multilayer on Ti-811 alloy surface via ion-assisted magnetron sputtering deposition (IAD) technique to enhance the fretting damage of this material. The $\mathrm{Cu} / \mathrm{Ni}$ film could significantly improve the fretting wear and fretting fatigue resistance of Ti-811 at room temperature because its excellent comprehensive properties with well toughness, high strength and good lubrication action. The studies of Zhang [ㄱ, ]ㅡ, Jin [] ] and Du [2] indicated that the lubrication coating were beneficial to improving the wear resistance of materials by reducing its friction coefficient. Thus, the soft foils of copper, nickel and PTFE with well lubrication were helpfully to reduce the friction coefficient of fretting wear on titanium alloy surface. It was beneficial to enhance the fretting fatigue life of this alloy. Simultaneously, compared with IAD technique, the copper foil, nickel foil and PTFE lamella are more economical to resistant the fretting damage of titanium alloy at room temperature.

In this work, the fretting wear and fretting fatigue resistance of Ti-6AL-4V alloy were reported. The soft interlayer of copper foil, nickel foil and PTFE lamella were used to reducing the fretting damage of this alloy. Some fretting wear and fretting fatigue tests have been performed in order to clarify the role of those inter-layers during the fretting damage processes of titanium alloy.

\section{Experimental procedures}

The FF samples and fretting pads were obtained from Ti-6Al-4V alloy bars (F16 mm). Additionally, The FW samples and fretting pads were obtained from Ti-6Al-4V alloy bars (F30 mm) and Ti-6Al-4V alloy bars (F16 mm), respectively. This material contains 6.1 Al, 3.9V, 0.2Fe, 
$0.10 \mathrm{C}, 0.14 \mathrm{O}, 0.003 \mathrm{H}$, and balance Ti. The microstructure of this annealing titanium alloy is an equiaxial a-phase and intergranular b-phase. This titanium alloy had a surface micro-hardness of $380 \mathrm{HK}_{0.245 \mathrm{~N}}$. The other mechanical properties of this material are: $\mathrm{s}_{0.2}=1010 \mathrm{MPa}, \mathrm{s}_{\mathrm{b}}=$ $1080 \mathrm{MPa}, \mathrm{d}=15 \%$ and $\mathrm{y}=41 \%$. The copper foil (micro-hardness: $131 \mathrm{HK}_{0.098 \mathrm{~N}}$ ), nickel foil (micro-hardness: $277 \mathrm{HK}_{0.098 \mathrm{~N}}$ ) and PTFE lamella with $50 \mathrm{~mm}$ thickness were put between the titanium alloy specimens and fretting pads respectively aims to prevent the fretting damages of this alloy from FF and FW. Thus, four contact types has been studied in this work, they are: Ti-6Al-4V alloy - Ti-6Al-4V alloy (TT), Ti-6Al-4V alloy - copper foil - Ti-6Al-4V alloy (TCT), Ti-6Al-4V alloy - nickel foil - Ti-6Al-4V alloy (TNT), and Ti-6Al-4V alloy PTFE lamella - Ti-6Al-4V alloy (TPT).

The FF resistance of copper foil, nickel foil and PTFE lamella were determined via GPS-100 high-frequency fatigue machine (Fig. 1) under room temperature. The load mode of the fatigue samples was pull-pull. The contact state between the fatigue samples and the pads was cylinder to camber [10]. Relative fretting displacement between the fatigue specimen and the pads was produced by the different elastic deformation of them. The contact stress of the pads with the specimen was $85 \mathrm{MPa}$, which was applied by a stress ring tester. The other parameters of FF test were: maximal load of $550 \mathrm{MPa}$, stress ratio of 0.1 , frequency of $115.0 \mathrm{~Hz}$. The value of FF life was obtained by the average life of three samples. The morphologies of fracture surfaces and wear regions were evaluated via Scanning electron microscopy (SEM).

The FW test were conducted using a FW devise installed in a SDS100 servo-hydraulic machine [11]. The displacement and fretting frequency between the plane samples and the cylinder pads was controlled by this fatigue machine at $80 \mathrm{~mm}$ and $20 \mathrm{~Hz}$, respectively. In addition, the normal load was applied by a stress ring tester and was controlled at $100 \mathrm{~N}$. The worked region of the fretting pads had a length and roughness of $4 \mathrm{~mm}$ and $0.05 \mathrm{~mm}$, respectively. The tangential force and the number of cycles were record via a computer. The friction coefficients and the morphologies of wear surface after 50000 cycles were used to evaluate the FW property of this Ti-6Al-4V alloy samples. The maximum friction coefficient calculated by $m=Q_{\max } / \mathrm{N}\left(\mathrm{Q}_{\max }\right.$ : the maximum tangential force between the plane sample and the fretting pad; $\mathrm{N}$ : normal load), and the morphologies of wear surface were studied via SEM. 


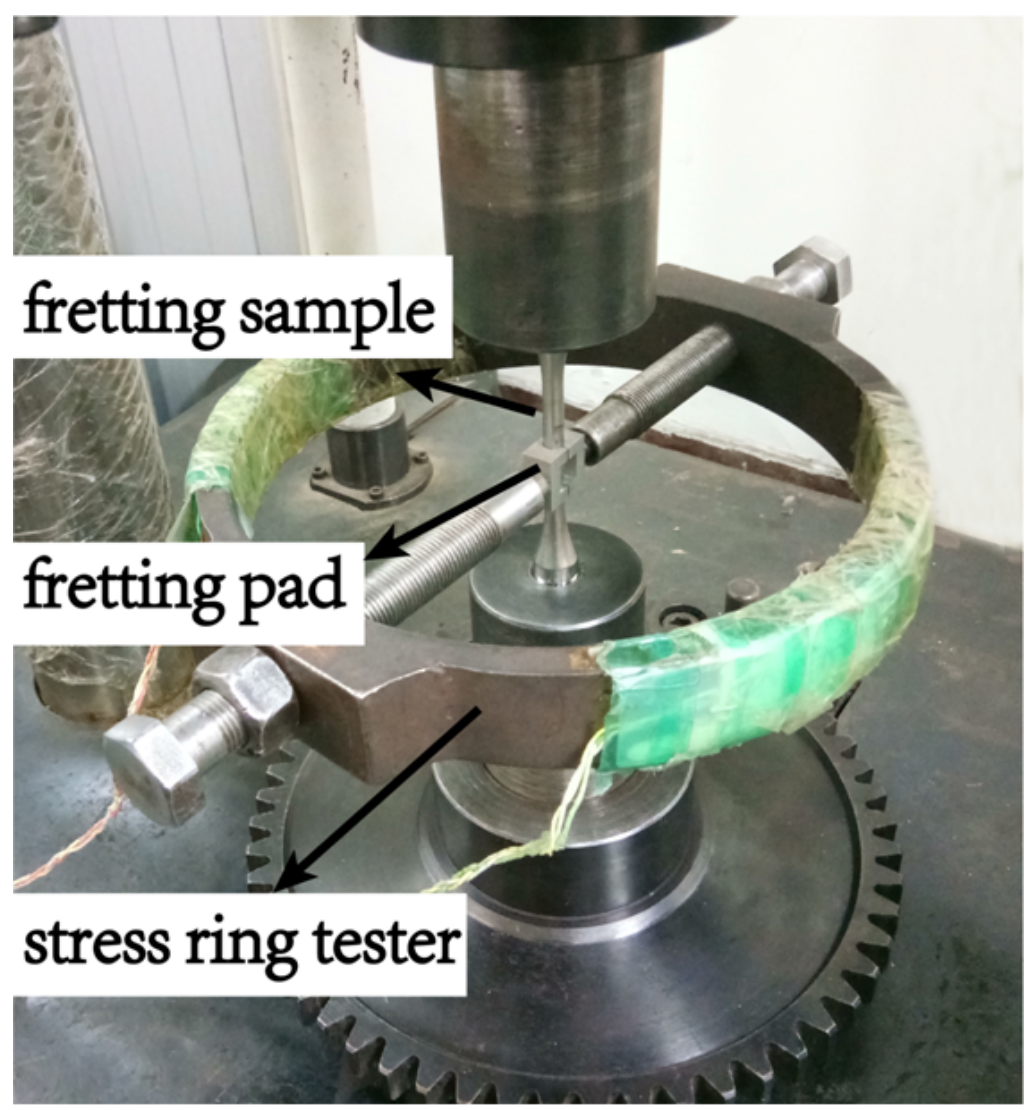

Fig. 1 The picture of fretting fatigue test

\section{Results and discussion}

\subsection{Friction coefficients}

FW testing was carried out to evaluate the fretting wear resistance of titanium alloy under different contact conditions. The friction coefficient of components is an important factor to estimate its fretting wear performance. Fig. 2 shows the variations in friction coefficient of titanium alloy with the cycle of numbers under the different conditions. The friction coefficient of titanium alloy was about 1.1. This high friction coefficient was ascribed to: on one hand, the Ti-6Al-4V alloy was very sensitive to adhesive wear [12]. The relative displacement and wear frequency between the fatigue sample and the fretting pad is so small and high, respectively. Additionally, the thermal conductivity of titanium alloy is low. The titanium alloy sample was very easy to bond with the counterpart under the high normal loading during the process of fretting wear test. On the other hand, the wear debris would be oxidated in air and very hard to discharge during fretting wear. The oxide with higher hardness than titanium alloy could lead a serious abrasive wear damage on the surface of titanium alloy. The two aspects all could produce a high friction coefficient.

The friction coefficient of copper foil and nickel foil contacted with titanium alloy was approximate, and lower than titanium alloy. It indicated that the copper foil and nickel foil could reduce the friction coefficient of Ti-6Al-4V alloy. Thus, the fretting wear resistance of titanium alloy could be enhanced via copper foil and nickel foil, which was ascribed to the two interlayers reduced the adhesive and abrasive wear damage of Ti-6Al-4V alloy. Simultaneously, the fretting fatigue life of titanium alloy was effectively improved by PTFE lamella. The friction coefficient of titanium alloy was reduced by 10 times via PTFE lamella. It was effectively to improve the fretting fatigue life of titanium alloy by reducing the 
fretting wear damage of this material. However, the lasting property of this PTFE lamella was lower than copper foil due to its low loading capability.

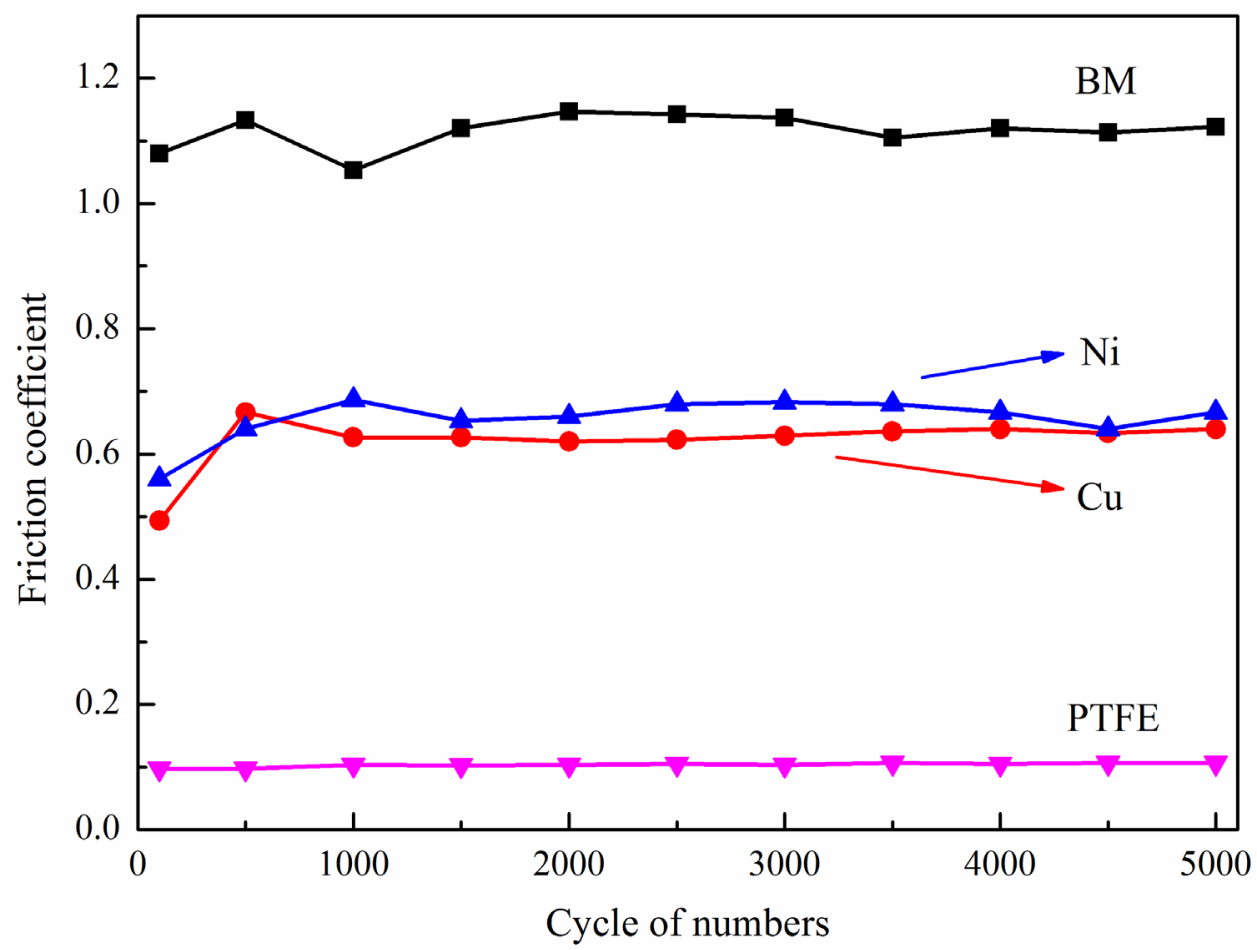

Fig. 2 Friction coefficients of Ti-6Al-4V alloy with different conditions

\subsection{Fretting fatigue life}

Fig. 3 shows the fretting fatigue life of Ti-6Al-4V alloy samples evaluated in different condition. It indicated copper foil, nickel foil and PTFE lamella could significantly improve the fretting fatigue life of Ti-6Al-4V alloy. The order of enhancement in the FF resistance of the titanium alloy was copper foil $>$ PTFE lamella $>$ nickel foil. The fretting fatigue life of this alloy with copper interlayer was 5 times higher than the base material, which could be ascribed to the low hardness and well toughness of copper foil as a cushion on the worn surface to fatigue specimen and counterpart. Simultaneously, the low friction coefficient of copper foil (Fig. 2) is good for relieving the tangential force of fretting wear damage between the fatigue sample and the pad during the fretting fatigue test. However, the effectiveness of the harder nickel foil was lower on reducing the normal load applied by the fretting pads, compared with the copper foil. It indicated that the combined action of well toughness and excellent fretting wear resistance of interlayer is beneficial to improve the fretting fatigue resistance of materials. 


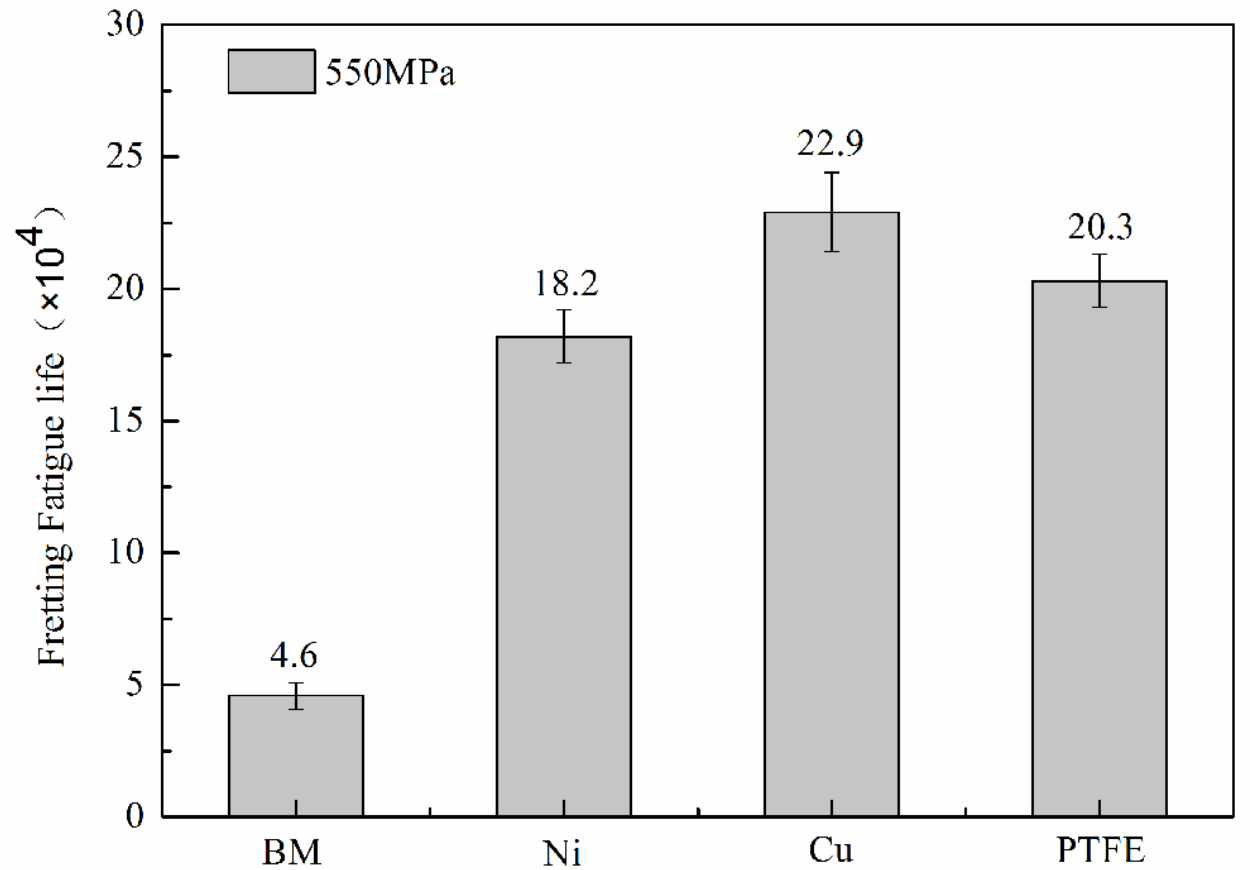

Fig. 3 The fretting fatigue life of Ti-6Al-4V alloy in different conditions

\subsection{Fracture surface topographies}

The fatigue fracture topographies of Ti-6Al-4V alloy are shown in Fig. 4, the (b), (d), (f), and (h) are zoom images of fatigue source area under TT, TCT, TNT, and TPT contact conditions, respectively. All of the fatigue fractures displayed three typical zones (Fig. 4 (b), (d), (f) and (h)): the crack initiation zone (region 1), the crack propagation zone (region 2), and the instantaneous fracture zone (region 3). The blue arrows in the zoon images (Fig. 4 (b), (d), (f) and (h)) are fatigue crack initiation location. All of the fatigue cracks initiate from the surface at some stress concentration regions, such as the bottom of some surface defect zone produced by fretting wear. Additionally, the fracture surfaces of titanium alloy under TT and TNT contact condition both present the characteristics of multisource. However, the fracture surfaces of titanium alloy under TCT and TPT contact condition both present the characteristics of a single fatigue crack source. It indicated that the rate of fatigue crack initiation on TCT and TPT conditions was lower than TT and TNT conditions. This is a one reason to improve the fretting fatigue life of titanium alloy via copper foil and PTFE lamella.
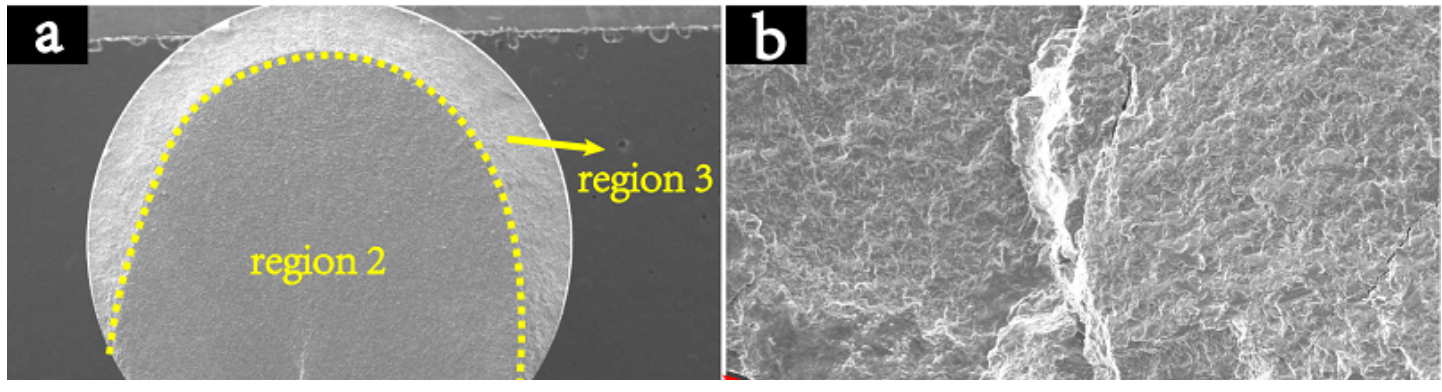

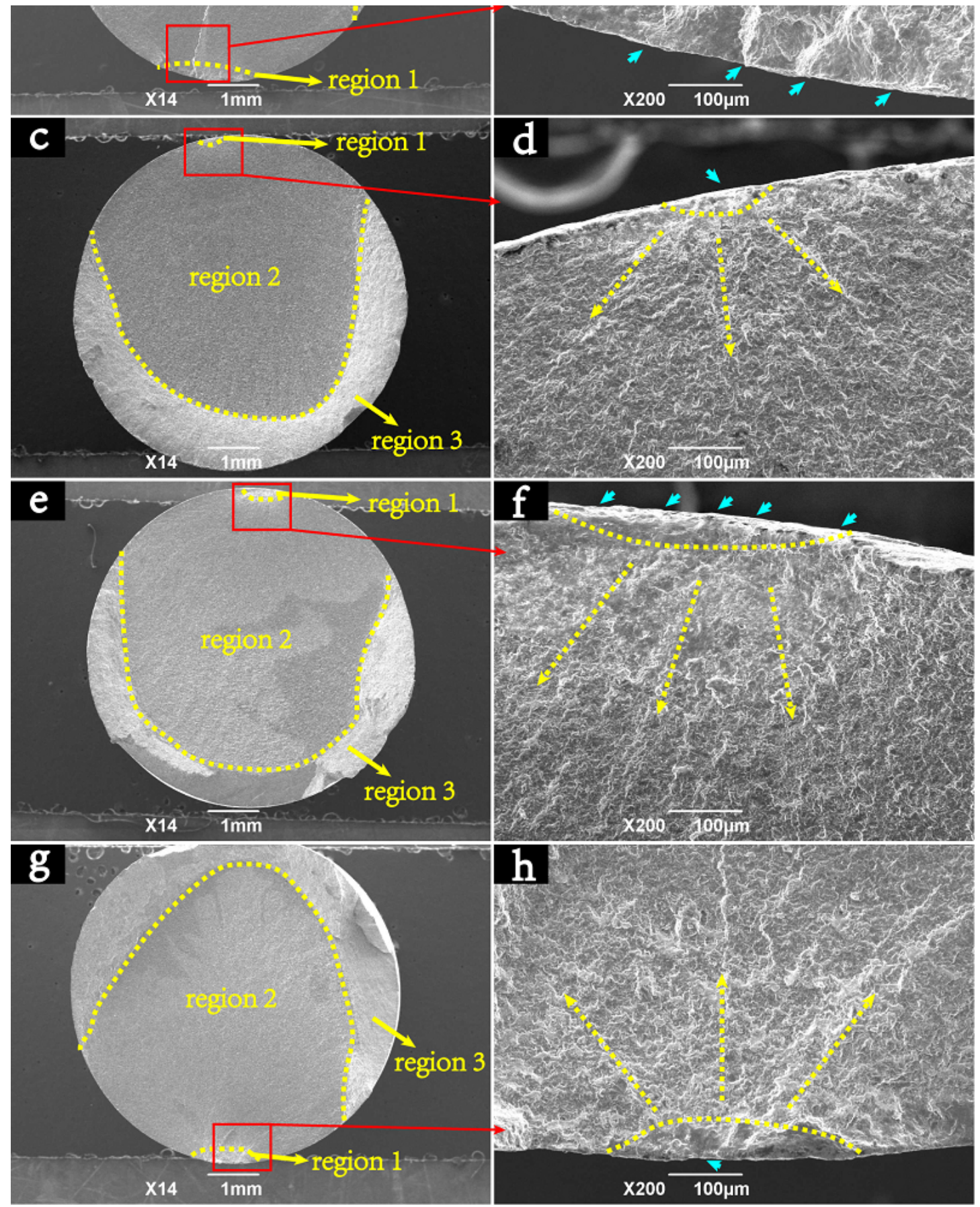

Fig. 4 Fracture morphologies of Ti-6Al-4V alloy with different conditions: (a) (b) TT, (c) (d) TCT, (e) (f) TNT, (g) (h) TPT. 


\subsection{Wear damage of contact region on titanium alloy}

Fretting wear damage is an important reason to produce fretting fatigue fracture of materials. Fig. 5 shows the morphologies of wear region on the titanium alloy surface after fretting fatigue test. The wear form of titanium alloy base material was abrasive wear, adhesive wear and oxidative wear. The copper, nickel and PTFE interlayer could significantly improve the fretting wear resistance of titanium alloy due to the reduction of abrasive wear. Ti-6Al-4V alloy with copper foil, nickel foil and PTFE lamella had higher fretting fatigue life than titanium alloy without interlayer, which was ascribed to the well fretting wear resistance of titanium alloy in those contact condition.

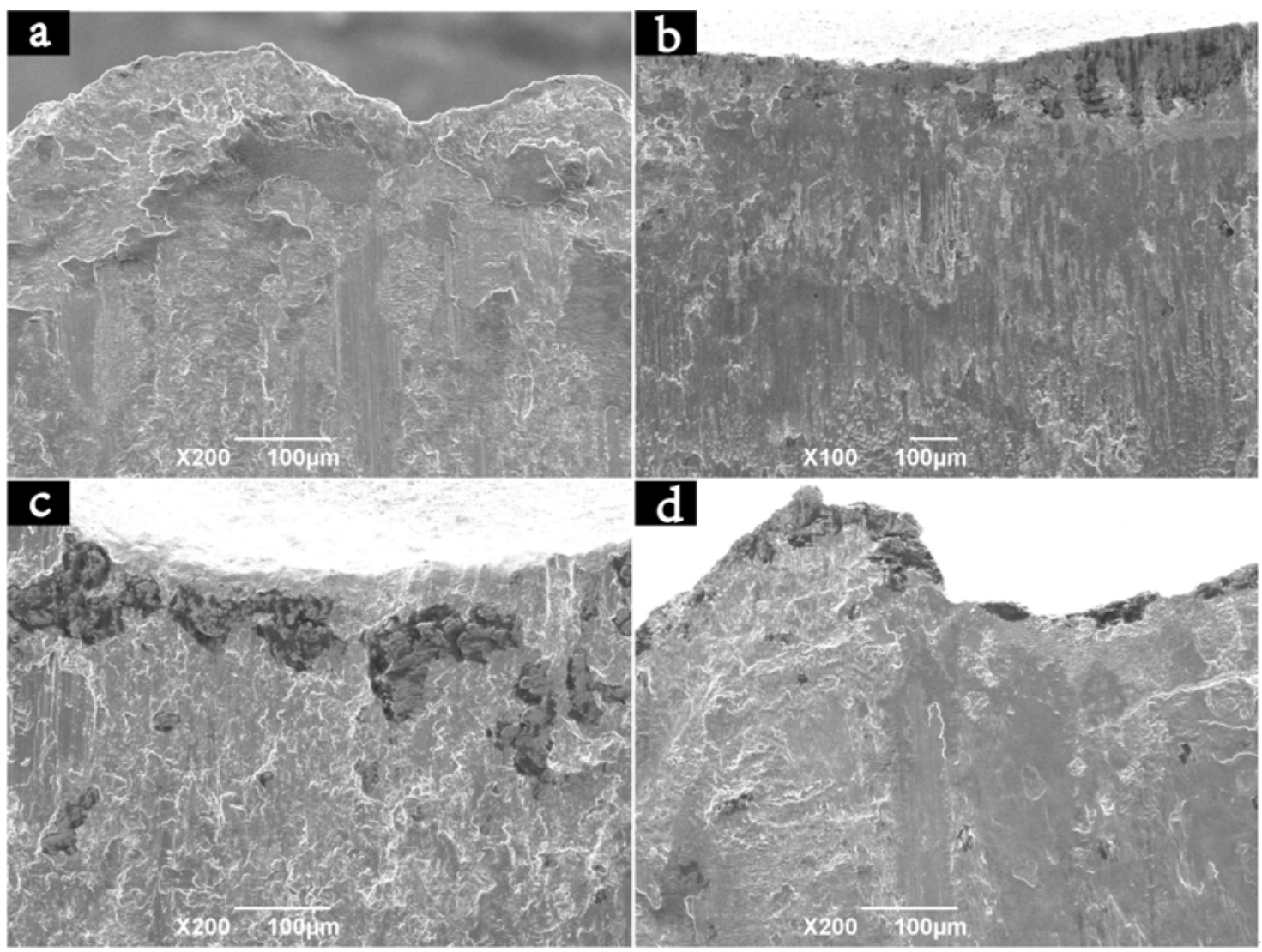

Fig. 5 Contact region SEM images of Ti-6Al-4V alloy under different contact conditions: (a) TT, (b) TCT, (c) TNT, (d) TPT.

\subsection{Wear morphologies of inter-layers}

The wear morphologies of copper foil and nickel foil are shown in Fig. 6. The copper and nickel foils were worn out after the fretting fatigue test. However, the fracture form of copper foil and nickel foil was different. The copper foil has an obvious shear slip crack. This crack makes the titanium alloy pads straightly contact with titanium alloy fatigue samples, and then introduce a seriously wear damage on the surface of this specimen to lead a fatigue fracture. Nevertheless, a shape of fold occurred on the surface of damaged nickel foil, which was bad for the fretting 


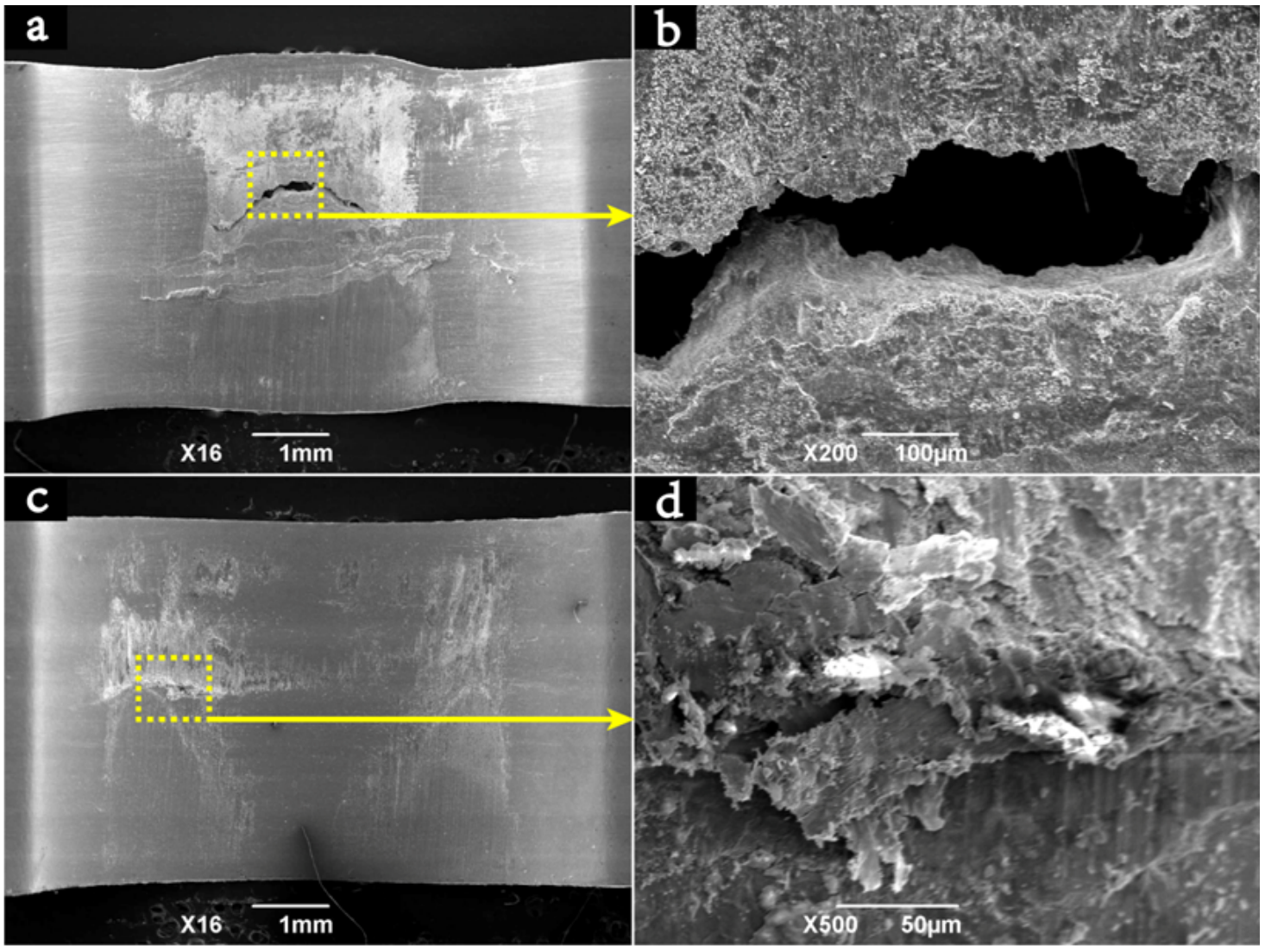

Fig. 6 Wear morphologies of copper ((a), (b)) and nickel ((c), (d)) interlayers after fretting fatigue test

\section{Conclusions}

The improvement of copper foil, nickel foil and PTFE lamella on the fretting wear resistance and fretting fatigue resistance of Ti-6Al-4V alloy were investigated in this work. On the basis of the experimental analysis, the main results were as follows:

(1) The friction coefficient of PTFE lamella was about 0.1. The lowest friction coefficient of PTFE, compared with copper foil, nickel foil and substrate, was of great benefit to improving the FF property of Ti-6Al-4V alloy

(2) Additionally, the fretting fatigue life of Ti-6Al-4V alloy was significantly enhanced via copper foil, it was 5 times higher than base material. The excellent performance of the copper foil was attributed to its more toughness, well loading capability, suitable hardness and low friction coefficient. Besides, the nickel foil could also improve the FF property of this alloy slightly due to its lower friction coefficient, compared with substrate. 
(3) Under the contact conditions of TCT, TNT and TPT, all of the fretting fatigue fractures of Ti-6Al-4V alloy were due to the primarily fracture of the interlayer.

(4) Overall, the FF and FW properties of the Ti-6Al-4V alloy were improved by copper foil, PTFE lamella and nickel foil. The method of only reducing the friction coefficient of the titanium alloy has limited potential to enhance its FF resistance. Therefore, it is necessary to get suitable hardness, toughness, well loading capability and low friction coefficient via using soft interlayer for high FF resistance.

\section{Acknowledgements}

This work was supported by the National Natural Science Foundation of China (Nos. 51771155)

\section{$\underline{\text { References }}$}

[1] J.F. Lamethe, P. Sergot, A. Chateauminois, B.J. Briscoe, Wear, 255 (2003) 758-765.

[2] D. Du, D.X. Liu, Z.Y. Ye, X.H. Zhang, F.Q. Li, Z.Q. Zhou, L. Yu, Appl Surf Sci, 313 (2014) $462-469$.

[3] K. Miyoshi, H.S.B. Jeffrey, C.H. Hager, J.S. Zabinski, R.L.V. Wal, R. Andrews, K.W. Street, B.A. Lerch, P.B. Abel, Tribol Int, 41 (2008) 2433.

[4] C.H. Hager, J.H. Sanders, S. Sharma, Wear, 265 (2008) 439-451.

[5] O.J. McCarthy, J.P. McGarry, S.B. Leen, Int J Fatigue, 62 (2014) 180-193.

[6] C.H. Hager, J. Sanders, S. Sharma, A. Voevodin, Wear, 263 (2007) 430-443.

[7] X.H. Zhang, D.X. Liu, G.H. Liu, Z.Y. Wang, B. Tang, Tribol Int, 44 (2011) 1488-1494.

[8] X.H. Zhang, D.X. Liu, X.Y. Li, H.S. Dong, Y.T. Xi, Materials, 10 (2017).

[9] O. Jin, S. Mall, J.H. Sanders, S.K. Sharma, Surf Coat Tech, 201 (2006) 1704-1710.

[10] D.L. Weidong Zhao, Xiaohua Zhang, Ying Zhou, Ruixia Zhang, Hao Zhang, Chang Ye, Int J Fatigue, 121 (2019) $30-38$.

[11] C.B. Tang, D.X. Liu, B. Tang, X.H. Zhang, L. Qin, C.S. Liu, Appl Surf Sci, 390 (2016) 946-958.

[12] S. Basseville, G. Cailletaud, Wear, 328 (2015) 443-455. 